\title{
CARA - THE CENTER FOR ASTROPHYSICAL RESEARCH IN
} ANTARCTICA

\author{
D A Harper and John Bally \\ Center for Astrophysical Research in Antarctica \\ and University of Colorado
}

The Center for Astrophysical Research in Antarctica is a new National Science Foundation Science and Technology Center formed to explore and exploit the unique advantages of the Antarctic Plateau for astrophysical observations.

Member institutions currently include the University of Chicago, Princeton University, Boston University, the University of Illinois, Haverford College, George Williams College, AT\&T Bell Laboratories, Rockwell International Corporation, and Adler Planetarium. Administrative offices of the Center are located at the University of Chicago's Yerkes Observatory in Williams Bay, Wisconsin.

Currently planned research includes the following projects:

- AST/RO (Antarctic Submillimetre Telescope and Remote Observatory) will use a $1.7 \mathrm{~m}$ diameter submillimetre telescope to survey the Galactic plane, the Galactic Center, and the Magellanic Clouds in the emission from the fine-structure line from CI at $609 \mu \mathrm{m}$ and the $\mathrm{J}=4 \mathrm{P} 3$ line of $\mathrm{CO}$ at $650 \mu \mathrm{m}$.

- The COBRA (Cosmic Background Radiation Anisotropy) experiment will search for and map anisotropies in the cosmic background radiation on angular scales ranging from 15 arcmin to $20^{\circ}$ at sufficient sensitivity to place stringent constraints on current theories of the origin of the universe.

- SPIREX (South Pole Infrared Explorer) will use a near-infrared telescope and infrared array detectors to explore the potential of the Antarctic Plateau as an infrared site and to make deep $2.4 \mu \mathrm{m}$ continuum surveys for faint sources including primeval galaxies, brown dwarf stars and dust-obscured objects in regions of star formation.

Center researchers and a broadly-based group of astronomers from the United States and other countries will conduct experiments to measure environmental parameters including sky brightness, atmospheric transmission, and seeing. They will also discuss and develop plans for more powerful telescopes which could be built in the future at the South Pole or other Antarctic sites.

\section{FRENCH PLANS FOR ASTRONOMY IN ANTARCTICA}

\section{J L Puget, Institut d'Astrophysique Spatiale}

French astronomers have been involved in research programs in Antarctica since 1979 when a successful campaign on helioseismology was done by a collaboration between the Nice University and the Bartol foundation. Several campaigns followed on this subject. In 1984 sub-mm observations of the diffuse galactic emission were done by a collaboration between the LPSP (Verrières le Buisson) and the Bartol foundation. These showed the high quality of the site for such observations not only because of the low water vapour content, which gives good transparency, but also because of the very low sky noise.

Future plans for Antarctic research by French astronomers are not well defined yet but strong interest exists in three fields: 